\title{
Experimental Results on the Process of Goal Formation and Aspiration Adaptation
}

\author{
Reinhard Selten \\ Department of Economics, \\ Institute for Empirical Research in Economics, \\ University of Bonn, Adenauerallee 24-42, 53113 Bonn, Germany \\ rselten@uni-bonn.de
}

\begin{abstract}
We experimentally investigate how subjects deal with a multi-period planning and decision problem. The context is a profit maximization task in a computer-simulated monopoly market over fifty time periods. The subjects are provided with a computerized short-run planning tool allowing them to check feasibility of any aspiration level for any set of feedback variables of the respective planning period. Our results fall into two categories, first, regarding the selection of goal variables and, second, regarding the process of aspiration adaptation. As to the former category, we find that subjects with at least median success change their goal variables less frequently than those below median success. Relatedly, goal persistence, a measure of a subject's tendency to stick to the current goal system, is strongly positively correlated with success. As to the latter category, we find that aspiration levels tend to be changed in strong agreement with basic principles of Aspiration Adaptation Theory (Sauermann and Selten 1962, Selten 1998, 2001). In addition, we find that in many cases the process of aspiration adaptation leads into a nearly stationary situation in which the aspiration level does not significantly change over several periods. Those subjects who reach a nearly stationary situation tend to be more successful and more goal persistent than those who do not. Some subjects who reach a nearly stationary situation deviate from Aspiration Adaptation Theory in order to find a more profitable nearly stationary situation.
\end{abstract}

